\title{
WOUND HEALING AFTER OPERATIONS ON THE EXTRA-OCULAR MUSCLES OF MONKEYS*
}

\author{
BY \\ R. M. INGRAM $\dagger$ \\ From the John E. Weeks Institute for Ophthalmology, the University of Oregon Medical School, \\ Portland, Oregon, U.S.A.
}

TENOTOMY of the extra-ocular muscles and their re-attachment to the sclera is an essential part of operations for strabismus and retinal detachment. Investigation of the processes whereby these wounds heal is desirable for two reasons:

(1) The tendon or muscle does not always become re-attached at the intended site and this may account, in part, for the unpredictability of strabismus operations. Opinions differ as to how these operations should be performed, and there is a need for sound principles.

(2) Improved techniques in detachment surgery offer better hopes of cure. Nevertheless, re-operations are frequent and they are complicated by adhesions formed after earlier procedures involving the extra-ocular muscles. The cause of these adhesions needs to be established.

This particular paper concerns tissue repair after strabismus operations in monkeys. The problem has been studied in rabbits by Carroll and Blake (1932) and by me (Ingram, 1965a). However, the findings in rabbits cannot be directly applied to man because the muscle-to-sclera junction is dissimilar. Sub-human primates are more suitable experimental animals since, in both monkeys and man, a tendon joins the muscle to the sclera.

These studies may incidentally contribute to our knowledge of the healing of wounds in tendons elsewhere in the body, because similar tissues are involved and consideration must be given to what is already known about this subject. The process of tendon repair has been linked in the past with the study of transplanted tendon grafts, and interest has centred around the viability of the grafted tissue rather than the method by which the graft was joined to the host. Peer (1955) has reviewed the conflicting views on the subject of tendon repair. Some workers believed that regeneration of the tendon took place by outward growth from the cut ends of the tendon. Others believed that the new tendon was formed mainly from the sheaths surrounding the tendon stumps. In between these two schools of thought, Mason and Shearon (1932) stated that the union between the two ends was first effected by proliferation of the tendon sheath and that, after a few days, the tendon

* Received for publication December 31, 1964.

† Present address: Royal Eye Hospital, Manchester, England. 
cells multiplied and eventually formed the new tendon. The problem centres round the origin of the fibroblasts that lay down collagen to join the two ends of the tendon. Do they arise from the fibrocytes in the tendon, from the connective tissue surrounding the wound, or do they come from both sites? One should remember that the morphological origin of the fibroblast is still uncertain (Grillo, 1964).

Incision and suturing of a tendon must necessarily damage the surrounding connective tissue, and repair will follow in both the tendon and the connective tissue. The observation that the two tissues appear to be intermingled during this process does not necessarily mean that the connective tissue reaction is essential to the healing of the tendon. The cornea is in some respects similar to tendon, and Weimar (1958) has shown that a corneal wound in the rat heals without intimate apposition with loose connective tissue. Of the fibroblasts in this wound 35 per cent. originated from the fibrocytes in the cornea, and 65 per cent. from the migration of monocytes from elsewhere.

The relationship between the orbital connective tissue and these wounds is of practical as well as theoretical importance. Swan and Talbot (1954) and Swan (1956) recommended that Tenon's capsule should be accurately re-sutured into its correct anatomical position after strabismus operations. Pierse (1962) believes that this minimizes the adhesions that occur after these operations.

It was decided, therefore, to study the healing of the wounds after strabismus operations in primates, with particular reference to the relative parts played by the regeneration of the tissues involved, especially the tendon fibres, muscle, and the adjacent connective tissue. Two series of operations were carried out:

(1) Recession of the lateral rectus combined with tenotomy and re-attachment of the medial rectus, using two different methods of suturing.

(2) Recession of the medial rectus combined with reefing of the lateral rectus.

\section{Experimental Materials and Trial Operations}

Adult Macaca rhesus monkeys were used, their average weight being $3.7 \mathrm{~kg}$. They were fed on a diet consisting of $250 \mathrm{~g}$. of purina monkey chow, which contains 15 per cent. crude protein, together with fat and various minerals and vitamins, pineapple, and half a banana daily.

Anaesthetic.-Young (1963) found that 1-(1-phenylcyclohexyl) piperidine (Sernylan) was suitable for retinoscopy and fundus examination in monkeys. Under this anaesthetic the eyes stared forwards, almost motionless, but some tone remained in the muscles. A dosage of $1.5 \mathrm{mg}$. per kg. body-weight was used in these experiments, but it was found that the effect of the drug wore off before the end of the operation on the second eye. Repeat doses of Sernylan proved unreliable, one animal going into opisthotonos, but supplementary intravenous or intramuscular injections of barbital enabled the operation to be completed satisfactorily.

Trial Operations.-Some trial operations were performed and the major technical difficulty was found to be the insertion of the sutures into the sclera. The sclera was very thin behind the original insertions of the tendons $(0.08 \mathrm{~mm}$., or one-fifth that of man, as measured by Chamberlain, 1954) and the incidence of perforation was high. This was overcome if the lateral, rather than the medial, rectus was recessed (there being better exposure of the wound area on this side of the eye). The use of atraumatic needles was important because 
the slightest pressure on the needle pushed the cutting edge, on the under side of some needles, through the sclera.

During resection of the medial rectus there was a feeling of tightness when the strabismus hook was under the tendon. This was partially relieved by gently pushing the hook back into the orbit and separating fascial attachments. Nevertheless, there seemed to be considerable tension on the sutures when only $3 \mathrm{~mm}$. were resected, and it was felt that the risk of the sutures tearing out of the muscle would be reduced if the tendon was detached and re-sutured to the original insertion without any portion being resected.

\section{Plan of the Experiments}

Because of these technical problems, the first series of operations consisted of lateral rectus recession and medial rectus tenotomy and re-attachment. It is recognized that this did not demonstrate the processes of healing after resection, but the tissues involved in the wound following tenotomy and re-attachment of the medial rectus are similar to those involved in a resection of $5 \mathrm{~mm}$. or less of the lateral rectus and to those in retinal detachment operations in man.

Experience gained during this series made it possible to recess the medial rectus without perforating the sclera in a second series of operations. Tenon's capsule proved to be a complicating factor in the study of healing after the operations on the medial rectus in the first series, and so it was excised from the wound area on this occasion. In order to clarify observations on the parts played by the tendon and peritendinous tissue respectively, the lateral rectus tendon was reefed.

\section{Method of Operation}

In the first series of operations, both eyes of each animal were operated upon. Conjunctiva and Tenon's capsule were incised parallel to, and approximately $4 \mathrm{~mm}$. from, the limbus. The tendons were exposed, care being taken to avoid injuring them. The lateral rectus was recessed $3 \mathrm{~mm}$. The medial rectus was tenotomized and re-attached as described.
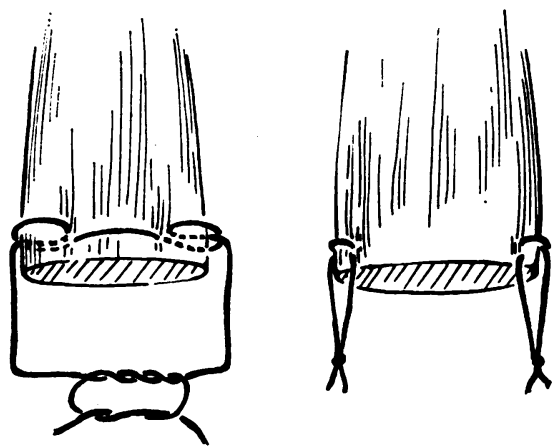

FIG. 1.-Diagram illustrating the position of the sutures in the wounds relative to the end of the tendon-one double-armed suture (left) and two single-armed sutures (right).

Plain catgut $5 / 0$ sutures with atraumatic needles were used. Since it has been suggested (Ingram, 1965a) that the position of the sutures in the wound area might affect the regeneration of the tendon, one double-armed suture was used to fasten the tendons to the sclera in each wound on the left eye, and two single-armed sutures were used in each wound on the right eye (Fig. 1). The conjunctiva was closed with a continuous $6 / 0$ plain catgut suture. The wound in Tenon's capsule was not sutured. 
In the second series of experiments the right eye only was used. The conjunctiva over the medial rectus was incised parallel to the limbus, and the proximal edge was gently lifted up and undermined with scissors. Tenon's capsule was opened in the same direction and separated from the tendon and sclera. A large tongue of Tenon's capsule was therefore dissected free and excised. The medial rectus was recessed $3 \mathrm{~mm}$., using two separate $5 / 0$ plain catgut sutures. The conjunctiva was closed with a continuous $6 / 0$ plain catgut suture.

The lateral rectus was exposed by incising conjunctiva and Tenon's capsule parallel to the limbus. A strabismus hook was passed under the tendon and one $5 / 0$ plain catgut suture was placed in the extreme edge of each side of the tendon $5 \mathrm{~mm}$. from its insertion. The needles were then passed through the superficial layers of the sclera level with, but above and below, the tendon's insertion. They were positioned in such a way that when the sutures were pulled tight and tied, the tendon was folded upon itself and the maximum possible width of the centre of the tendon would be seen in the microscopic slides without the complicating factor of having the suture in the section. Care was taken during this procedure to avoid injuring the tendon and as a result a somewhat inadequate fold was sometimes obtained. The conjunctiva was sutured with $6 / 0$ plain catgut.

In the first series of experiments one monkey was killed on each of the following days after operation: $1,2,3,4,5,7,10,14,21,28,56$, and 84 . Both eyes were removed for histological study. In the second series the right eye was removed on each of the following days after operation: $1,3,5,7,9,11,14,21,28,56$, and 84 .

\section{Method of Obtaining the Specimens}

The usual methods of enucleation could not be used for two reasons: (1) dissection of the muscles would distort the picture in the wound area; and (2) it was important to maintain the conjunctiva and Tenon's capsule in their natural positions relative to the wounds for the maximum possible distance behind the limbus.

The following method, which is partly enucleation and partly evisceration was, therefore, developed. A horizontal incision, about $1 \mathrm{~cm}$. long, was made through the centre of the cornea with a Bard-Parker knife. This incision was extended across the limbus with two cuts and continued through the sclera and overlying tissues on either side of the wound. The conjunctiva, right out at the canthus, was button-holed with sharp scissors, which were then pushed through the hole down to the periosteum and spread. Dissection was then continued into the orbit in a plane immediately inside the periosteum. The two incisions down each side of the wound were also continued backwards, a tongue of tissue being thus dissected out. The sclera was cut in the region of the optic nerve from inside the eye. This exposed the appropriate muscle and enabled it to be cut far back in the orbit. The specimen was gently lifted out of the orbit, any remaining strands of tissue being cut with scissors. The procedure was more satisfactory if it was done immediately after the animal had been exsanguinated.

In order to prevent contracture, the specimen was immediately pinned out on a piece of cork and immersed in 10 per cent. formalin. It was embedded in paraffin, and sections were cut from four levels across each wound. Haematoxylin and eosin and haematoxylin and van Gieson stains were used.

\section{Histological Study of Lateral Rectus Recession Wounds}

For two days after operation the wound area was oedematous and infiltrated with leucocytes, principally around the sutures. The tendon and its nuclei appeared normal. A prominent feature was a thin layer of inflammatory reaction along the 


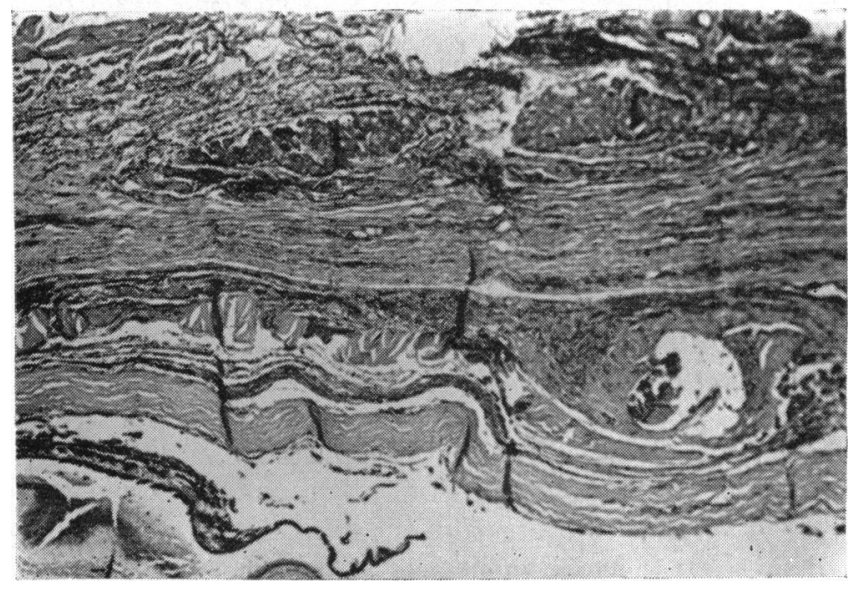

FIG. 2.-Lateral rectus recession. Day 1. This shows the inflammatory reaction in the peritendinous layer of connective tissue. H. and E. $\times 20$.

superficial and deep surfaces of the tendon (Fig. 2). This extended from the suture 3 to $4 \mathrm{~mm}$. back into the orbit, almost as far as the muscle, which was hardly affected by the operation. Tenon's capsule was a well-defined layer, quite separate from the underlying tissues and apparently unaffected by the operation. On the third day the pattern of healing had become apparent. The inflammatory reaction around the suture was appreciably less. The tendon fibres stained well. The peritendinous inflammatory reaction consisted of blood vessels, inflammatory cells, and some fibroblasts. There was no cellular reaction around the original insertion of the tendon. Again, Tenon's capsule was a separate, well-defined layer extending over the whole wound area. There is some evidence in the wounds examined on the second and third days after operation that the muscle was damaged to a minor degree, and there were more than the normal number of nuclei in the muscle fibres on the third day. By the fourth day there was very little inflammatory reaction around the sutures. New collagen had been deposited between the end of the tendon and the suture, but there was still no direct attachment of the tendon to the sclera. Many of the fibroblasts in the wound area seemed to come from the peritendinous inflammatory layer. The normal appearance of the fibrocytes immediately behind the abrupt end of the tendon (Fig. 3) made it unlikely that they had been a significant source of the fibroblasts.

The first signs that the tendon was becoming re-attached to the sclera were seen on the fifth day. Collagen had been deposited in the connective tissue on the deep surface of the tendon where this merged with the wound area. Sections stained with van Gieson showed that this collagen was immature, and the attachment did not appear firm. There was a clear line of demarcation between the end of the tendon and the connective tissue in the wound area, and the fibroblasts appeared to have migrated from the small blood vessels in the wound area and in the adjacent peritendinous inflammatory layer. The absence of any regular pattern to the collagen fibres deposited around the sutures was a point of distinction from the reaction seen in these wounds in rabbits (Ingram, 1965a). In the two wounds examined on the 


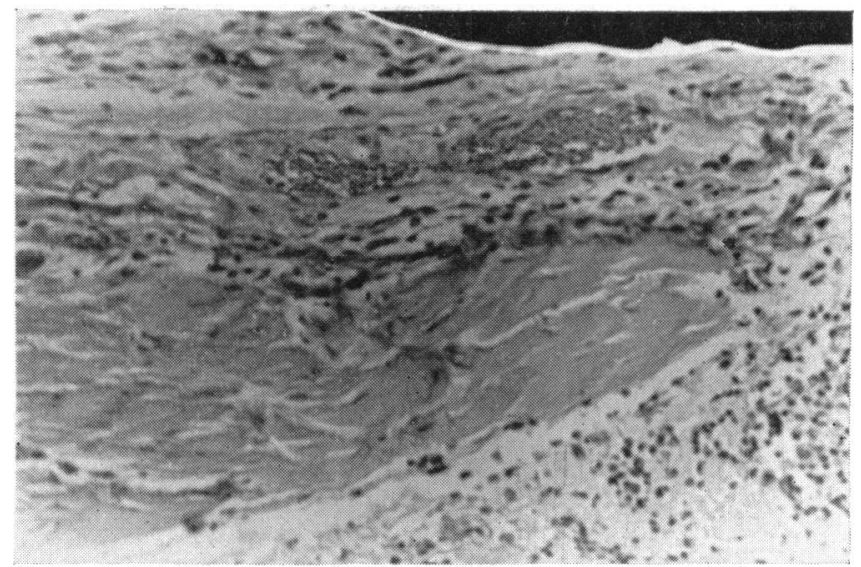

FIG. 3.-Lateral rectus recession. Day 4. This shows that the cells are connected in the connective tissue surrounding the end of the tendon, which appears almost inert. H. and E. $\times 120$.

fifth day, Tenon's capsule had merged with the tissues underneath it. On the seventh day, the tendon appeared to be more securely attached to the sclera. There was only a minimal ring of fibrous tissue around the suture. Tenon's capsule was adherent to the tendon-to-sclera wound, but it was separated from the peritendinous inflammatory layer behind the wound and from the loose connective tissue in front of this. New connective tissue was interwoven between the ends of the tendon fibres.

The re-attachment appeared firmer ten days after operation and it extended for 1 to $2 \mathrm{~mm}$. behind the suture (Fig. 4). The collagen was still immature and deposited irregularly in the wound area. Tenon's capsule was clearly demarcated from the moderate inflammatory reaction around the suture. The intermingling of the tendon fibres with the newly deposited collagen was more advanced. The fibrocytes in the tendon were enlarged and contained nucleoli. They looked like fibroblasts, but they did not appear to be more numerous than in normal tendon. It seems more

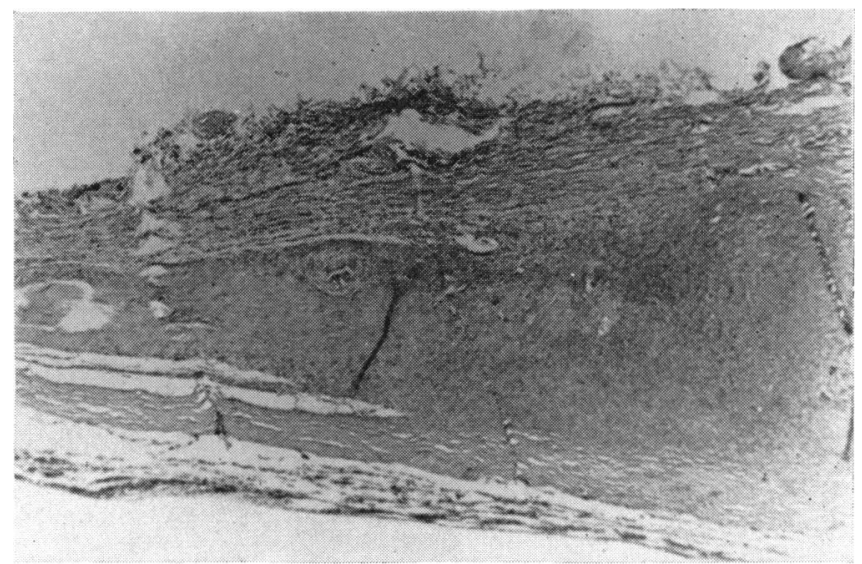

FIG. 4.-Lateral rectus recession. Day 10. New collagen laid down in the wound area is becoming interwoven with the free end of the tendon. H. and $\mathrm{E}, \times 20$. 
likely that the connective tissue had grown into the end of the tendon, rather than out from the tendon fibres. By the fourteenth day, the wound was becoming organized. The tendon fibres were more intimately intermingled with the newly deposited collagen fibres which now assumed a definite anteroposterior direction. The peritendinous inflammatory zone, containing many fibroblasts and a few blood vessels, stained more palely than the tendon itself, and it gradually tapered off as the tendon was followed back into the orbit (Fig. 5). Loose connective tissue containing many fibroblasts was present between the suture and the original insertion. Tenon's capsule passed superficial to, and separate from, the underlying tissues, but there was a distinct condensation of fibrous tissue on its deep surface. This formed an organized layer extending from behind the wound area to the original insertion. The possibility that this is the beginning of a "pseudotendon" must be borne in mind (see Discussion).

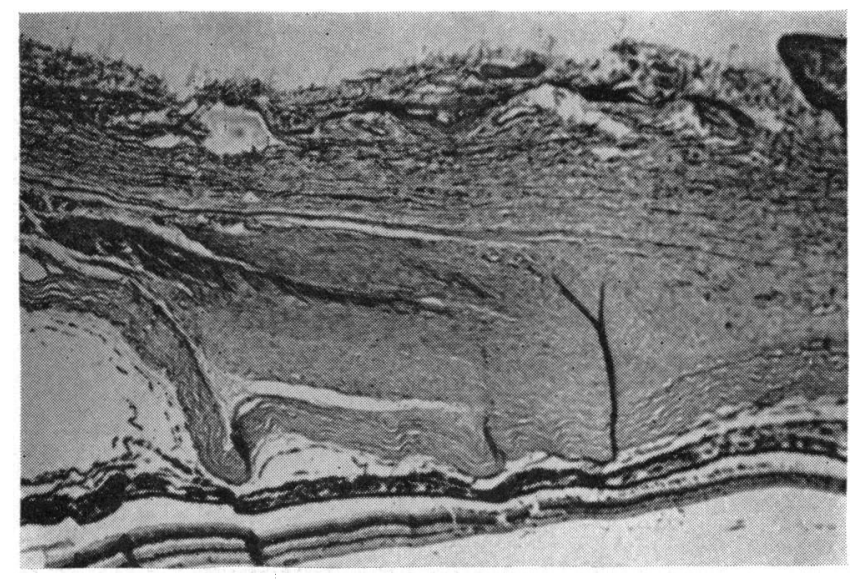

FIG. 5.- Lateral rectus recession. Day 14. The tendon is firmly attached to the sclera. Note the position of the new collagen in relation to the peritendon. Tenon's capsule is quite separate from the underlying wound. H. and $\mathrm{E} . \quad \times 20$.

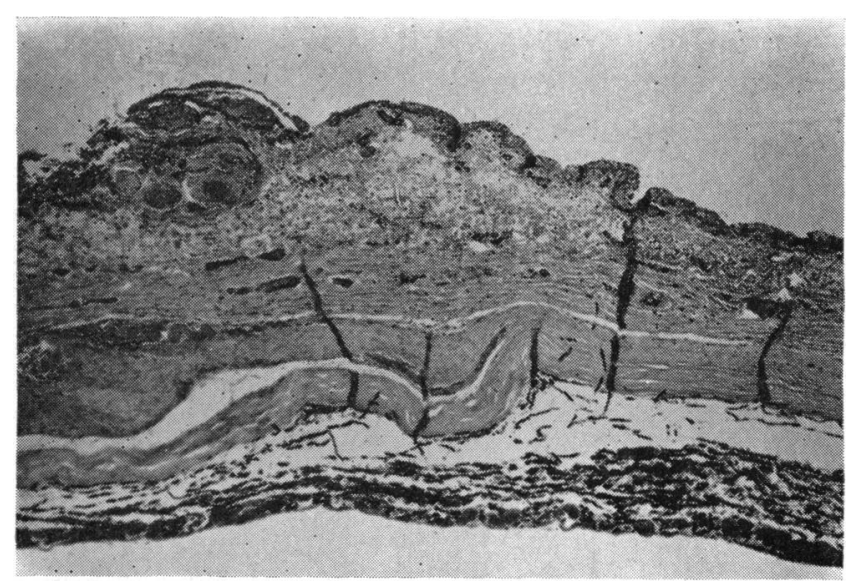

FIG. 6.-Lateral rectus recession. Day 21. A recognizable tendon now joins the muscle to the sclera. Note how this attachment extends forward closely adherent to the sclera. H. and E. $\times 20$. 
The organization of the re-attachment was further advanced three weeks after operation (Fig. 6) and by this time the catgut sutures had disappeared, leaving behind only a small collection of lymphocytes. The collagen fibres were arranged in an orderly manner and attached the tendon to the sclera over a wide area extending anteriorly almost up to the original insertion. New fibrous tissue extended back from the wound area to surround the tendon, continuous with the peritendinous connective tissue. The fibroblasts in this tissue were larger and more numerous than those in the tendon, suggesting that they were actively involved in the process of repair. By the twenty-eighth day, the wound was comparatively neat and orderly. Fairly mature fibrous tissue joined the tendon to the sclera over a wide area which extended almost up to the original insertion.

The fibrous tissue which formed the re-attachment was not completely mature two months after operation; it was less compact, had a higher cellular content, and stained slightly paler than normal tendon. It extended $2-3 \mathrm{~mm}$. in front of the original site of the recession. A thin layer of dilated blood vessels represented the remains of the peritendinous connective tissue zone behind the wound. In one wound, Tenon's capsule was separate from the fibrous tissue in the wound area, but adherent to the tendon behind and to the original insertion in front. It is possible that, in this eye, it formed a pseudotendon. Three months after operation the new collagen had matured, and it was difficult to differentiate it from the underlying sclera. The tendon was attached to the sclera by three strips of fibrous tissue (Fig. 7). The middle one of these is probably the real attachment which had become organized so

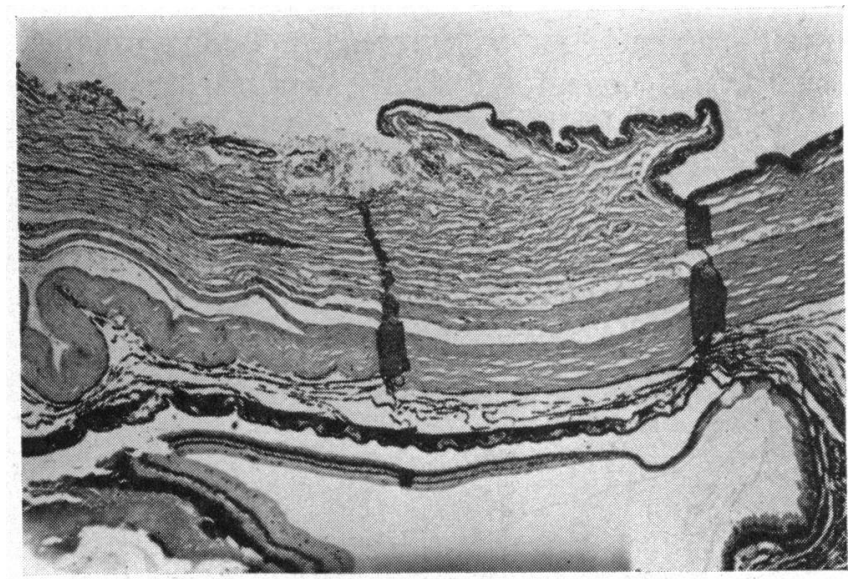

Fig. 7.-Lateral rectus recession. Day 84. This shows three separate strips of fibrous tissue joining the tendon to the sclera. $\mathrm{H}$. and $\mathrm{E}$. $\times 20$.

that it looked like true tendon. The strip of fibrous tissue on its deep surface may have arisen from the peritendinous connective tissue in this situation, but the superficial layer, of uncertain origin, represents a pseudotendon in the sense that it is an extension of fibrous tissue ahead of the new insertion. It could be the remains of the peritendinous connective tissue zone which existed on the superficial surface of the tendon and which extended forward toward the old insertion, or it could be a condensation of the deep layer of Tenon's capsule. 


\section{Histological Study of Medial Rectus Re-attachment Wounds}

The principal features of these wounds on the first two days after operation were oedema and an inflammatory reaction around the sutures. Portions of tendon and the remains of the original insertion were entangled in the sutures. Apart from there being less inflammatory reaction and oedema the picture had changed little by the third day; $2 \mathrm{~mm}$. of tendon were seen behind the suture in one wound, and a definite peritendinous connective tissue layer was seen on both surfaces of this. No tendon or peritendon was seen in the other wound. There was an increased number of nuclei in the damaged muscle fibres. Some collagen had been deposited in the wound area by the fourth day. The tendon fibres were disorganized, but they stained normally and there were no changes in their nuclei. The cut edges of Tenon's capsule were adherent to the muscle and the inflammatory reaction surrounding the suture. The more extensive dissection of the tendon that was necessary during these operations and the proximity of the muscle to the wound would account for there being more tissue damage than was seen in the lateral rectus recession wounds over the same period of time.

The first real signs of a fibrous re-attachment of the muscle to the sclera were seen on the fifth day. In both these wounds, the muscle was 3-4 $\mathrm{mm}$. behind the suture, possibly because the suture had slipped. The area between the muscle and the suture was filled with irregularly stained collagen which gradually merged into Tenon's capsule superficially. In one eye, Tenon's capsule had actually slipped into the wound. On the seventh day, more collagen had been laid down in the wound area, but the wounds from both eyes appeared less orderly than the recession wounds at this time. Oedema and the acute inflammatory reaction had subsided, but the cut edges of Tenon's capsule were incorporated in the wound. The opening in Tenon's capsule was over the suture which had slipped $2-3 \mathrm{~mm}$. behind the original insertion. In another area Tenon's capsule was split and the deeper layer was adherent to the sclera in front of the site of the re-attachment of the tendon (Fig. 8).

By the tenth day the wound was becoming orderly. Again, Tenon's capsule had merged with the tissues deep to it. The collagen fibres between the original insertion

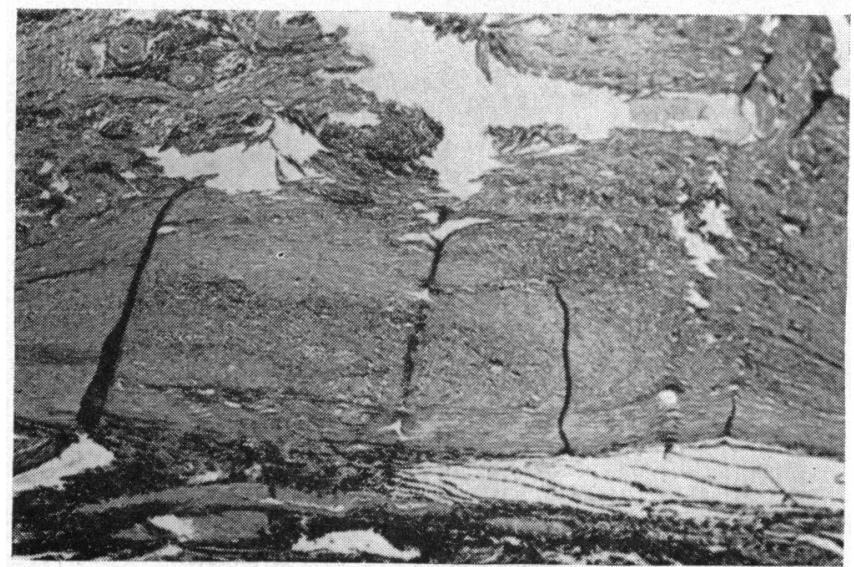

Fig. 8.-Medial rectus re-attachment. Day 7. Tenon's capsule incorporated in the wound. H. and E. $\times 20$. 
and the muscle $2-3 \mathrm{~mm}$. behind this were arranged very roughly in an anteroposterior direction. Sections stained with van Gieson indicated, however, that most of this collagen was immature.

Regenerating muscle fibres appeared to be growing forward into the collagen tissue which formed the re-attachment fourteen days after operation. Sheets of mature fibrous tissue in the wound area were surrounded by newly deposited collagen, but it was not clear whether these were strips of tendon which were entangled in the suture, or whether they were strips of Tenon's capsule which had prolapsed into the wound area. As in the recession wounds there were only minimal signs of a foreignbody type of granuloma around the sutures. There was still some reaction around the suture three weeks after operation, but the muscle was firmly re-attached to the sclera (Fig. 9). The proximal end of Tenon's capsule had retracted and come to lie

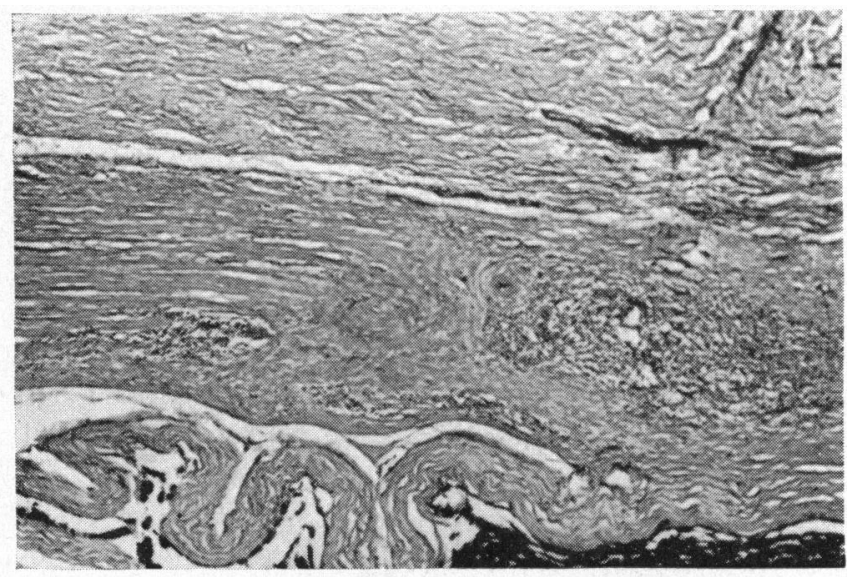

FIG. 9.-Medial rectus re-attachment. Day 21. This shows the fibrous re-attachment of the muscle to the sclera. H. and E. $\times 20$.

over the tendon-to-sclera wound. The collagen fibres lay irregularly between the edges of Tenon's capsule at an angle to the sclera, and the fibroblasts were spreading into the defect principally from a collection of mononuclear cells adjacent to the sclera (Fig. 10).

Organization of the fibrous tissue was slightly more advanced at twenty-eight days, but there was no appreciable difference until two months after operation. At this time, a definite tendon could be made out, and the picture in these wounds resembled that seen in the recessions, $3 \mathrm{~mm}$. or more of the tendon being recognizable. In one eye, a thin branch of fibrous tissue left the deep surface of the tendon and was attached to the sclera about $2 \mathrm{~mm}$. behind the original insertion. The fibrous tissue from this posterior insertion continued forward closely adherent to the sclera, and almost unrecognizable from it, to join the remainder of the tendon, which was attached at the original insertion. Tenon's capsule appeared quite normal, but between this and the tendon a third layer of well-organized and condensed fibrous tissue was seen. This seemed analogous to the superficial layer seen in this position in the recession wounds. There were several adhesions between it and the underlying tendon and posteriorly it was attached to the muscle. In the other eye only a thin tendon connected the muscle to the sclera at the original insertion. 


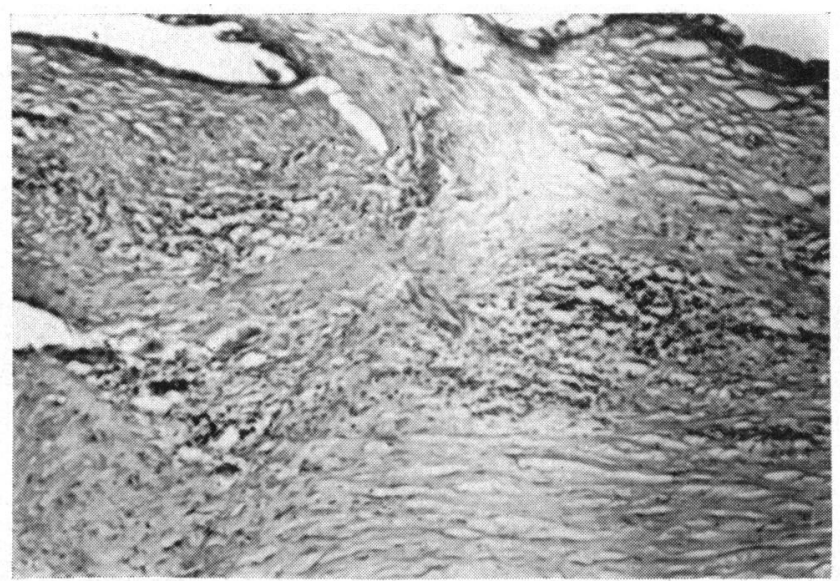

FIG. 10.-Medial rectus re-attachment. Day 21. Repair of the opening in Tenon's capsule by fibroblasts migrating into the defect from an adjacent collection of inflammatory cells. H. and E. $\times 60$.

At eighty-four days the tendon joined the muscle to the sclera at the original insertion. It was almost normal in appearance, but both the muscle fibres running into it and the tendon itself contained an abnormally high number of nuclei, indicating that the process of healing had not yet been completed. In the sections from the left eye, the tendon was split longitudinally into two. Many muscle fibres ran into the posterior end of both layers which closely resembled each other in structure. The split may have been an artefact, but at another level in the same wound there was a third layer on the deep surface of these, and this was attached to the sclera $1 \frac{1}{2}-2 \mathrm{~mm}$. behind the remainder of the tendon and continued forward, barely distinguishable from the underlying sclera (Fig. 11). At some levels in the wound in the other eye, the tendon was split up and attached to the sclera in several places over an area 2 to 3 $\mathrm{mm}$. behind the original insertion.

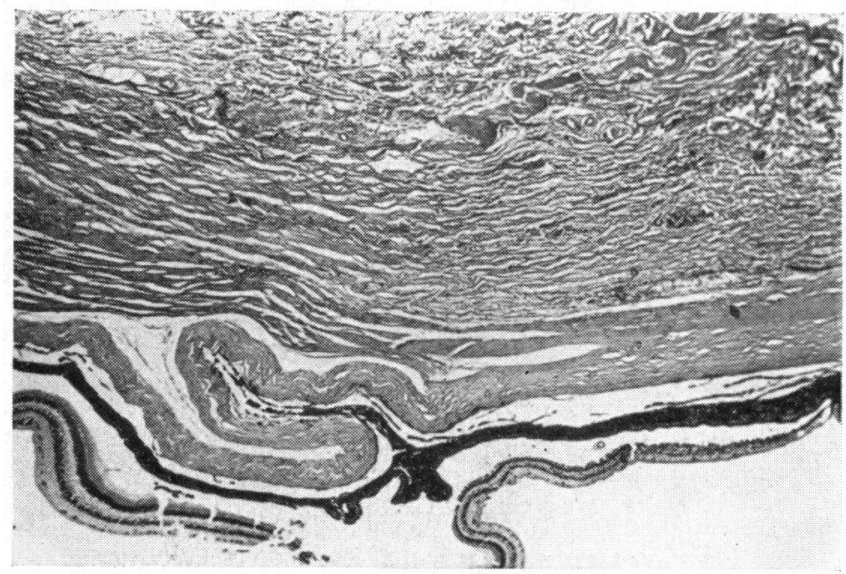

FIG. 11.-Medial rectus re-attachment. Day 84. A short length of tendon, split into three layers, joins the muscle to the sclera. H. and $\mathrm{E} . \quad \times 20$. 


\section{Histological Study of Medial Rectus Recession Wounds}

The wound examined on the day after operation was the only one to show that Tenon's capsule had not been effectively excised. However, on this occasion, the tendon was surrounded by a peritendinous connective tissue layer, exactly as was shown in the lateral rectus recession wounds. Sections from the wound examined three days after operation showed only adipose tissue superficial to the wound. The tendon was entangled in the suture and the muscle was right behind it. No peritendinous connective tissue layer was seen in this wound, but a moderate amount of damage had been inflicted on the muscle fibres which were infiltrated with macrophages. These cells were pleomorphic and their origin was a matter of speculation. Some of them appeared similar to the nuclei in the regenerating muscle fibres, but others could well have arisen from "resting" cells in the fatty connective tissue or have migrated into the area from blood vessels. Polymorphonuclear leucocytes and lymphocytes were confined to the area surrounding the suture, and there was no appreciable inflammatory reaction in the tissues superficial to the wound. On the fifth day, numerous palely staining pleomorphic cells containing one or two tiny nucleoli were spreading forward from the damaged muscle into the area surrounding the suture.

There was no real sign of any re-attachment until the seventh day. In part of this wound the tendon was seen behind the suture, but there was no clear-cut peritendinous zone. Cells (fibroblasts or myoblasts?) were streaming forward from the damaged muscle and spreading into the wound area. Macrophages (fibroblasts?) were migrating into the wound area from a few small blood vessels in the adipose tissue adjacent to the tendon immediately behind the suture. By the ninth day, the muscle was well attached to the sclera over a wide area which extended almost up to the original insertion. A suture was seen in two of the slides, but not in the others. Nevertheless, the same process appeared to have continued in both areas. Tendon was not seen in any of the sections, and the presumptive impression is that the fibroblasts came from either the muscle or the connective tissue adjacent to it, which, in this case, was not the same area as the peritendinous connective tissue layer. Sections from the seventh and ninth days showed that many fibroblasts had collected around the free distal end of Tenon's capsule, which was becoming adherent to the sclera around the original insertion. Quite a wide area of connective tissue reaction and fibrosis was involved. No signs of haemorrhage were seen.

There was little difference in the overall picture of the wounds examined eleven and fourteen days after operation, but by the twenty-first day the muscle was attached to the sclera by a well-orientated band of fibrous tissue which was adherent to the sclera for 2-3 mm. There were no adhesions between this fibrous re-attachment and the loose connective tissue superficial to it. Further back in the orbit, however, the muscle fibres were adherent to the connective tissue. Many fibroblasts surrounded the distal end of Tenon's capsule which was becoming bound down to the original insertion. The suture was not seen, but the remains of the inflammatory reaction surrounding it were still present.

The process of organization of the fibrous tissue continued through the sections examined one month after operation, until at two months about $1 \mathrm{~mm}$. of tendon 
joined the muscle to the sclera. Three months after operation the picture was similar except that there were many fewer nuclei in the muscle and the tendon. All the tissues in the wound area were much more normal at this time (Fig. 12). Two important observations were noted in these wounds. Firstly, there was no evidence of any "pseudotendon" in either. The muscle was attached to the sclera by only one strip of fibrous tissue, and not three, as after lateral rectus recession. Examination of 25 more sections from each wound confirmed this. Secondly, the re-attachment appeared to be almost linear in that there was no obvious forward extension of the fibrous tissue anteriorly along the sclera. There were, however, quite marked adhesions between Tenon's capsule and the sclera in front of the insertion of the tendon.

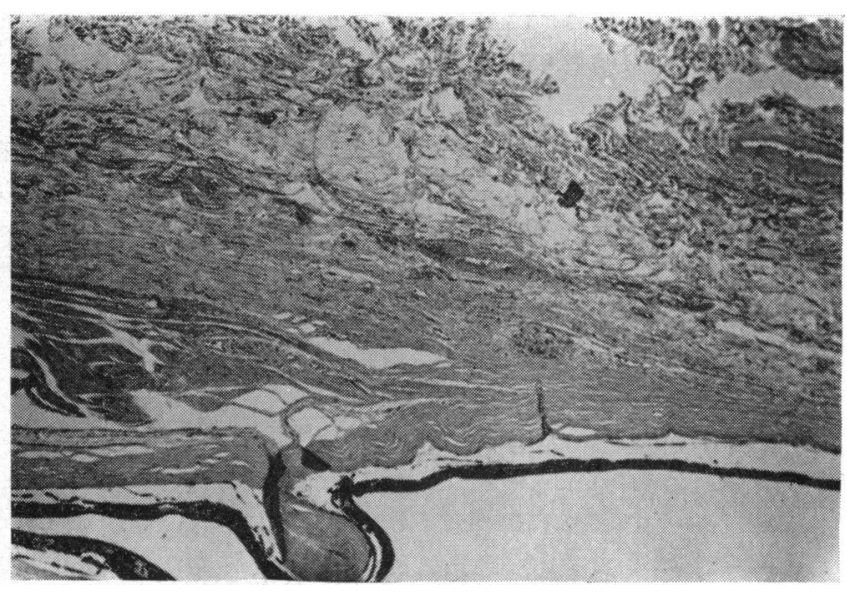

FIG. 12.-Medial rectus recession. Day 84. This shows the short strip of tendon joining the muscle to the sclera. H. and E. $\times 20$.

\section{Histological Study of the Lateral Rectus Reefing Wounds}

The wounds on the first and third days after operation showed only the signs of acute inflammatory reaction around the sutures and in the peritendinous connective tissue. On the fifth day, many fibroblasts were seen in this layer for several millimetres behind the tendon's insertion. The nuclei in the tendon were enlarged and contained nucleoli (Fig. 13), but there was no obvious increase in their number. No folding was seen in the wound on the seventh day, but the peritendinous connective tissue layer was packed with fibroblasts on both surfaces of the tendon. In one specimen, the suture had severed the tendon $3 \mathrm{~mm}$. behind its insertion. This defect was being repaired by fibroblasts from the peritendinous connective tissue layer and, at this point, the early stages of an adhesion between the tendon and the sclera were evident. A similar observation was noted on the ninth day. In this wound, a good fold was obtained and it was becoming bound down to the sclera. The fibrocytes in the tendon of the wound examined on the eleventh day were enlarged and they contained nucleoli, as they did on the seventh and ninth days. However, they were not increased in number except in localized areas related to the repair of an area of 
tendon damaged at operation. In many cases the fibrocytes were enlarged for several millimetres on either side of the suture and clearly not related to any injury of the tendon. Adhesions were formed between the tendon and the sclera behind the fold

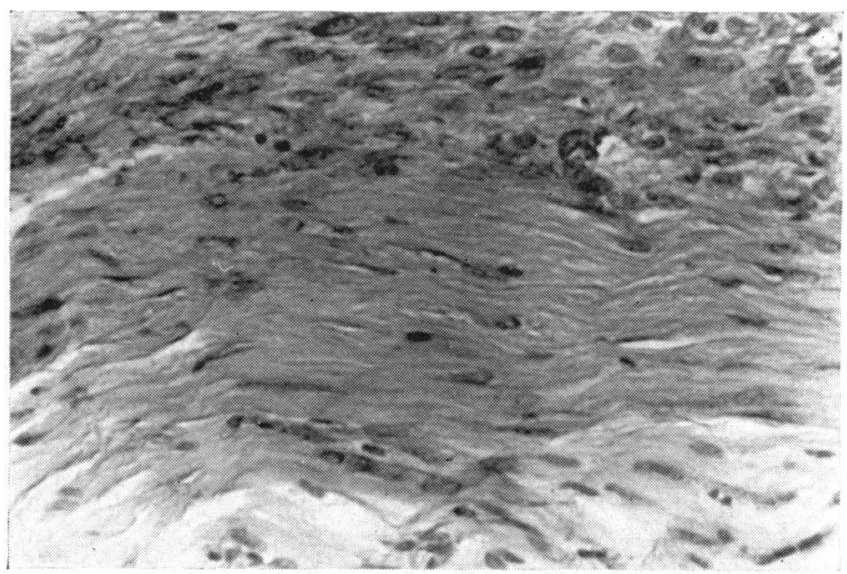

FIG. 13.-Lateral rectus reefing. Day 5. The nuclei in the tendon 2 to $3 \mathrm{~mm}$. behind the original insertion are enlarged and demonstrate nucleoli. Fibroblasts can be seen in the peritendon above the tendon. H. and E. $\times 172$.

in the twenty-one and twenty-eight-day wounds. In these wounds, the folded tendon was enveloped in a mass of new connective tissue which originated from the peritendinous connective tissue layer. The whole wound was remodelled by the time two and three months had elapsed after operation, and there was no evidence of any fold in either of these wounds, although adhesions were seen between the tendon and Tenon's capsule and between tendon and sclera for 3-4 $\mathrm{mm}$. behind the insertion, in both cases (Fig. 14).

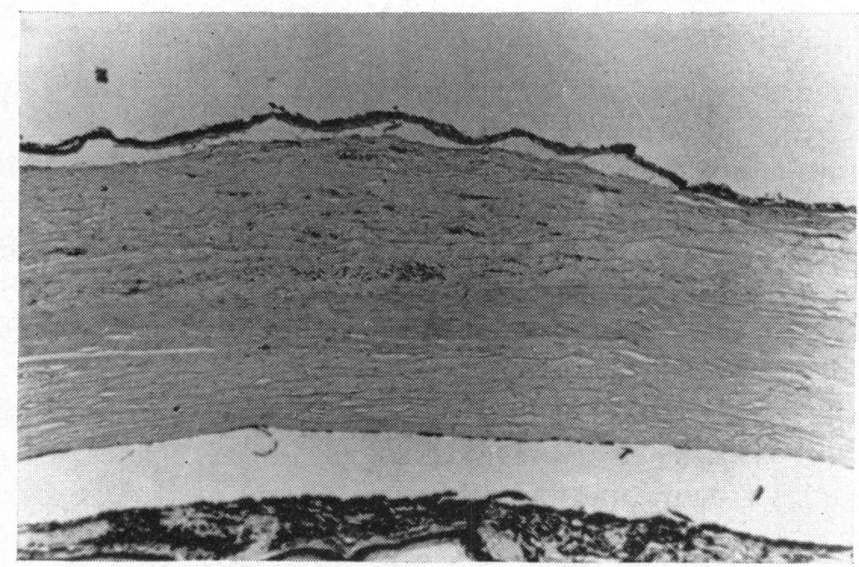

Fig. 14.-Lateral rectus reefing. Day 84. The area of the fold has been completely remodelled. Note the adhesions between the tendon and Tenon's capsule and the sclera on either side of it. H. and E. $\times 30$. 


\section{Discussion}

The second series of experimental operations was designed to demonstrate and clarify points raised in the study of the first series. The findings from the second series will, therefore, be considered later in this discussion.

Recession of the lateral rectus is commonly employed in the treatment of strabismus, but the choice of tenotomy and re-attachment of the medial rectus may be criticized because it does not show what happens when a large resection of muscle is performed. On the other hand, the medial rectus re-attachments demonstrated the healing process that would take place after moderate resections of the lateral rectus and after this muscle was re-attached during detachment operations. Recession of the lateral rectus gave a clear picture of the healing of an appreciable length of tendon on to the sclera and demonstrated the importance of the thin layer of connective tissue surrounding the tendon. Muscle fibres damaged at the time of operation participated in the repair process, but regeneration of new muscle did not affect the final point of re-attachment, as it did in rabbits.

The process of healing after lateral rectus recession presented an orderly sequence of events. The overall impression is that Tenon's capsule, the sclera, the original insertion, and the tendon all appeared to contribute little or nothing to the healing process. This is understandable because they are all basically the same in structure, namely, they are comparatively well-organized condensations of collagen fibres. It is not possible to exclude these structures as a source of the fibroblasts that entered the wound area, but it is unlikely that any was a major source. The fibroblasts probably originated in the loose connective tissue adjacent to the wound area, particularly the thin layer of connective tissue which immediately surrounds the tendon. Another function of this peritendon is repair of localized defects along the course of the tendon. This layer of connective tissue extends anteriorly to the limbus and posteriorly as far as the junction of the muscle with the tendon. It is the episclera which consists of numerous small blood vessels and a thin membrane. The fibroblasts could have originated from the blood cells within these vessels or from "resting connective tissue cells".

By comparison, the medial rectus re-attachment wounds showed a confused picture, and no pattern was discernible until two anatomical points were recalled. First, there was only a very short tendon between the medial rectus and the sclera. In some wounds this tendon was not seen, and in others much of it was entangled in the suture. This explains the frequent absence of anything resembling the peritendon that was such a prominent feature in the recession wounds. There was no clear-cut alternative source of the fibroblasts in these wounds. The second point concerns the anatomy of Tenon's capsule. Over the lateral rectus it is a well-defined, but thin, layer of collagen fibres, whereas over the medial rectus it is considerably thicker and it is less well condensed into a definite layer. Furthermore, it does not extend as far back into the orbit on the medial side as it does on the lateral side. In the lateral rectus recession operations, with one or two exceptions, Tenon's capsule remained as a distinct layer. It passed intact over the tendon-to-sclera wound, apparently taking no part in the cellular reaction underneath it. This was certainly not the case in the re-attachment wounds where it was often incorporated in the cellular reaction around the suture and adherent to the superficial surface of 
the muscle behind this. Tenon's capsule was opened parallel with, and directly over, the muscle insertion, and failure to close this wound appears to have complicated the healing process underneath.

If tendon wounds heal in the same manner, the findings from these experiments seem to indicate that the new tendon formed in the wound area would arise mainly (though not necessarily exclusively) from the fibroblasts originating in the adjacent connective tissue. Early workers did not separate the tendon sheaths from the tendon, but the ingenious experiments of Skoog and Persson (1954) effectively did this. They showed that the thin layer of connective tissue immediately surrounding the tendon, which they called "peritendon" (Fig. 15), was itself inadequate to permit healing within a normal period, and that the connective tissue outside this, which they called the "paratendon" was, in fact, the main source of the new connective tissue

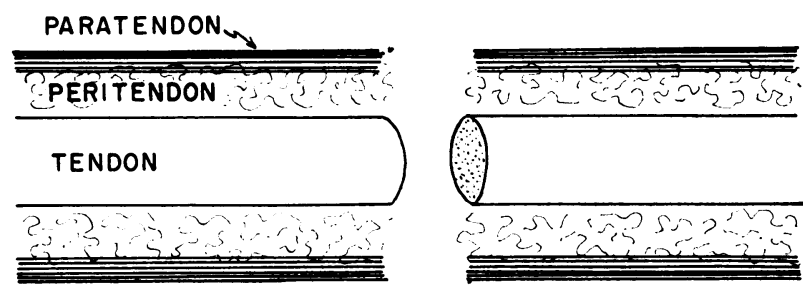

FIG. 15.-Diagram illustrating the positions of peritendon and paratendon (as named in this article) relative to tendon.

deposited between the cut ends of the tendon. They believed that the peritendon was essential to the normal nutrition of the fibrillary bundles of the tendon. When this layer was scraped off the tendon, there was a more pronounced connective tissue reaction which resulted in adhesions between the tendon and the surrounding tissues.

Examination of the photographs in Skoog and Persson's (1954) paper indicates that the peritendinous inflammatory zone, described in the present investigation, represents the peritendon of their experiments, and that Tenon's capsule is equivalent to their paratendon.

It seems, therefore, that there is some discrepancy in the conclusions drawn from the two investigations, but this is not so. In the medial rectus re-attachments there was virtually no peritendon (because there was such a short length of tendon behind the wound area), and paratendon (Tenon's capsule) was extensively damaged. The widespread intermingling of Tenon's capsule in these wounds does not necessarily indicate that Tenon's capsule (paratendon) was the source of the fibroblasts in the wound area. There is support for the belief that it does not fulfil this function because, firstly, Tenon's capsule is not a very vascular tissue, and, secondly, other sections from these wounds suggest that the wound in Tenon's capsule is itself healed by fibroblasts arising in the surrounding loose connective tissue. Skoog and Persson (1954) do not state whether the paratendon of the rabbit soleus is a well-organized layer of fibrous connective tissue like Tenon's capsule. If, on the one hand, it is a poorly organized vascular layer of connective tissue, then their findings could be explained on the grounds that removal of peritendon necessitated damage to a wide area of surrounding connective tissue involving paratendon. This would produce scar tissue formed in a wide area which included the tendon wound. On the other 
hand, if the paratendon of the rabbit soleus is a well-organized layer of connective tissue, then their findings would still be compatible with those of the present investigation, the difference being that the purpose of this connective tissue reaction would be to repair not only the tendon wound, but also the wound in paratendon, which itself would probably be more extensive because of the exposure necessary to remove effectively the underlying peritendon.

Skoog and Persson (1954) state that when paratendon was excluded from the wound area the "tissue reaction in the peritendinous connective tissue was of only moderate severity and performs no significant regenerative function during the first three weeks". Over the lateral rectus recession wounds, Tenon's capsule was relatively undamaged, and peritendon contributed sufficient collagenous tissue to heal this particular wound. It does not, however, necessarily mean that this would be sufficient in the case of a larger tendon, for example, the soleus of the rabbit. The difference in the findings could, therefore, be one of degree. One could postulate that a given amount of collagenous tissue would be formed from a given amount of connective tissue, and that the more collagenous tissue required, the more connective tissue must be involved in the wound area. The price paid for this is adhesions between the tendon and surrounding connective tissue, and this may be the effect of scarification as recommended by Gallie and LeMesurier (1924), Whiston and Walmsley (1960), and others. It would be neither necessary nor desirable in the case of healing strabismus wounds.

These experimental wounds were studied particularly from the aspect of regeneration of the tendon and the part which it might play in the final point of re-attachment to the sclera. Occasionally some of the fibrocytes in the tendon were enlarged and contained nucleoli. This was not noticed frequently, possibly because there was a moderate amount of folding of the tendon during preparation of the specimens. However, there was certainly no obvious increase in the number of fibrocytes in the tendon and there is nothing to indicate that the re-attachment is formed by an outgrowth from the tendon or the sclera.

Similarly, the wound in Tenon's capsule did not heal by an outgrowth of fibrous tissue from Tenon's capsule, but fibroblasts appeared to infiltrate it from the loose connective tissue on either side. It follows from this that, if the connective tissue reaction necessary to heal the wound in Tenon's capsule is adjacent to the tendon-tosclera wound, fibrous tissue deposited in one wound will become intermingled with that deposited in the other, and adhesions will be formed. Furthermore, if the edges of Tenon's capsule are widely separated, the area of connective tissue reaction and fibrosis necessary to repair this wound will be larger and hence the adhesions will be more widespread. It seems essential, therefore, to approximate the edges of the wound in Tenon's capsule, as suggested by Swan (1956). Pierse's (1962) suggestion that an intact Tenon's capsule forms a barrier preventing adhesions between the conjunctiva and the tendon-to-sclera wound is valid only if neither of these structures becomes adherent to it. Adhesions were among the factors discussed by Romaine (1961) as causes of motility problems after scleral buckling operations.

While it should be possible to restrict the formation of adhesions, it is quite a different matter to "control" the distribution of the fibrous tissue joining the free end of the tendon to the sclera. (At this stage the discussion concerns only the fibrous 
tissue which extends forward from the point of recession closely apposed to the sclera.) Since the tendon and sclera do not themselves form this re-attachment, it is unlikely that variations in these structures, e.g., the operation suggested by Worst (1962), will alter the final point of re-attachment either. Likewise, altering the method of suturing, and so the position of the sutures relative to the other tissues in the wound area, did not affect this. The absence of any marked granuloma formation around the sutures in these wounds may be explained by the different species reaction to catgut (Sewell, Wiland, and Craver, 1955). An alternative explanation could be that, whereas in the rabbit experiments $3 / 0$ plain catgut was used, the smaller $5 / 0$ plain catgut used in these experiments would produce less foreign-body reaction and a smaller granuloma. It is possible, therefore, that the use of a smaller suture has eliminated any effect that the position of larger sutures might have induced in the healing process.

Catgut sutures were selected for these operations in order that the complete series of events in the healing process could be seen in a reasonable time. In the rabbit experiments (Ingram, 1965a), the use of silk sutures did not alter the nature of events in the wound area. It merely delayed the completion of the process. It is unlikely, therefore, that the use of non-absorbable, as opposed to absorbable, sutures would affect the final point of re-attachment. It must be concluded that, in the present state of our knowledge, we cannot influence the healing process, so that a neat linear re-attachment of tendon to sclera is formed exactly where we desire it.

At the conclusion of the first series of operations, several points remained unanswered. Firstly, it is evident that the recessed tendon becomes re-attached over a wide area, often extending 2-3 mm. in front of the intended line on the sclera. Attention was also drawn to the formation of a "pseudotendon", but the origin of this was not established. Secondly, since the sclera and the original insertion played no significant part in the repair process after recession or re-attachment, the variable factor in the healing of strabismus operations is not the site of re-attachment, but rather the nature of the tissue that is re-attached to the sclera. In this respect, the length of tendon joining the medial and lateral recti to the sclera is different, and tissue repair following recession of the two muscles may not be the same. What, therefore, is the source of fibroblasts in wounds following operations where only a very short length of tendon or muscle is sutured to the sclera? What does happen when the medial rectus is recessed? Thirdly, confirmation was required for the observation that the fine peritendinous connective tissue layer was the important source of the fibroblasts in wounds involving the attachment of an appreciable length of tendon to the sclera. Changes were occasionally noted in the nuclei of the lateral rectus tendon, but the significance of this observation was not clear. Further evidence of the respective roles of peritendon and tendon was desirable, and it was felt that this could best be obtained by studying the effects of reefing the lateral rectus tendon and following the development of adhesions between the folded tendon and the sclera.

Experiments where the medial rectus was recessed after complete excision of Tenon's capsule strongly suggest that the muscle was the principal source of fibroblasts in these wounds. The loose adipose tissue in the orbit is not likely to be a major source when Tenon's capsule is intact. Haust, More, and Movat (1960) 
have shown that in blood vessels, smooth muscle cells can deposit collagen, and it is possible that the nuclei in the striated muscle fibres, which were damaged by the operation and liberated into the wound area, could also form collagen. The regenerating muscle nuclei assumed many and varied shapes, some of them resembling fibroblasts, but it is more likely that the fibroblasts migrated from the blood vessels in the connective tissue septa of the muscle. The distorted muscle fibres were so close together that the origin of these cells was not clear. This may be the important source of fibroblasts when resection of the other muscles results in the union of muscle to the sclera.

Many surgeons have been faced with the problem of operating upon muscles previously recessed or resected, but there are very few reports of operative findings on the position of the muscle and adhesions. Berens (1938) reported adhesions between the muscle and the sclera behind the insertion after resection. Gifford (1942) reviewed the literature and described two cases where the previously recessed muscle was found re-attached to its original insertion. In one of these cases adhesions between the muscle and the sclera were found behind the original insertion, but not in the other. In my clinical experience, the forward extension of the attachment of the recessed muscle has been variable, but occasionally a thick sheet of fibrous tissue joining the new insertion to the original insertion can be dissected out. This sheet of fibrous tissue is quite distinct from the fibrous re-attachment which extends forward adherent to the sclera, and which has already been discussed. The term "pseudotendon" could be applied to it because, although it is fibrous tissue and may function as tendon, it is not histologically identical with tendon (Ingram, 1963). The formation of this pseudotendon merits discussion for the following practical reasons: (1) its presence may alter any mechanical effect the recession may have; (2) when it is present, it may be possible to utilize it at any re-operation. It could be excised or recessed when the deviation remained under-corrected, or it could be advanced or resected in cases which had been over-corrected.

Following lateral rectus recession a pseudotendon was found in three out of four wounds, two and three months after operation. It was a condensed layer of fibrous tissue situated between Tenon's capsule superficially and the re-attachment of the tendon to the sclera underneath. On the basis of the findings in these wounds, it could be an organized layer of fibrous tissue laid down in the peritendinous connective tissue (episclera) on the superficial surface of the tendon extending anteriorly across the wound area. However, this layer was not prominent in the medial rectus re-attachment wounds, yet the re-attachment was formed by more than one strip of fibrous tissue as in the recession wounds. This suggested that the pseudotendon could be an organized layer of fibrous tissue which was formed in the deep layers of Tenon's capsule. This explanation supposes that these layers were damaged at the time of operation and were repaired from connective tissue which also formed the true re-attachment. The absence of any pseudotendons from the wounds in which the medial rectus was recessed and Tenon's capsule simultaneously excised, would seem to favour the second explanation, but it should be remembered that the peritendinous connective tissue was not a prominent feature of wounds following either operation on the short tendon of the medial rectus. There may, of course, be a combination of these factors to produce a pseudotendon. 
The findings from these wounds confirm earlier observations concerning Tenon's capsule. The wounds healed perfectly well in the absence of this tissue (paratendon). However, adhesions were formed between the free distal end of Tenon's capsule and the sclera in front of the recession wound. In this operation the two wounds healed separately as they were $2-3 \mathrm{~mm}$. apart, but this does not detract from the validity of conclusions drawn from these earlier observations. This operation enabled a closer study to be made of what happened to the free end of Tenon's capsule, and further supported the concept that the wound in this structure should be considered as a separate entity from the tendon-to-sclera wound. The excessive scar tissue formed around the free end of the gap in Tenon's capsule was striking. If these two wounds are adjacent to each other it is very likely that adhesions will be formed between them.

The lateral rectus reefing operations confirmed the important role of the peritendinous connective tissue as the source of the fibroblasts when there is an appreciable length of tendon behind the wound area. Furthermore, they clearly indicate that even minimum trauma to this thin layer produces adhesions between the tendon and the sclera behind its insertion. This could well occur after resection operations or after the re-attachment of tendons in operations for retinal detachment. Relatively gross trauma, such as tying a suture into the tendon or clamping it, is particularly conducive to the formation of adhesions.

It was interesting to find no suggestion of a fold two and three months after operation. My operation notes record that a satisfactory fold was obtained in both these eyes, but it is possible that the fold became unravelled. On the other hand, the prolonged turnover of collagen in a wound is well recognized (Harkness, 1961; Williams, 1957; Burns and Beighle, 1960) and it is possible that there was some remodelling of the fibrous tissue in the folded segment of tendon. If this were so, there would be no means of foretelling the amount by which a tendon could be shortened by reefing.

The role of the fibrocyte in the tendon is a major point of controversy in the mechanism of healing of tendon wounds. Skoog and Persson (1954) did not report any changes in these cells, but Mason and Shearon (1932) found that they contributed to the formation of new tendon after the first seven to ten days. The objection to this has always been the belief that whatever the origin of the fibroblast, it does not arise from the resting fibrocyte. Opposed to this widely held view is Weimar (1958), who was in the unique position of studying whole mounts of the rat cornea. This is the simplest medium for observing fibrocytes because corneal wounds are uncomplicated by changes in any surrounding tissue. She believes that the corneal fibrocytes do become fibroblasts, but only those within $0.2 \mathrm{~mm}$. of the wound edge.

Changes in the fibrocytes in tendons in these experimental operations were not seen before the fifth day, possibly because of the methods of preparation of the specimens. They were undoubtedly present from the fifth day onwards, particularly in the lateral rectus reefing wounds, thus confirming the observations of Mason and Shearon (1932). I do not, however, agree with the conclusions they draw-namely, that these cells contribute to the formation of collagen between the wound edgesfor two reasons: (1) the concentration of fibroblasts in the wound area was much greater than in the tendon, and there did not appear to be any increase in the number 
of fibrocytes in the tendon as the wound was approached; (2) there was clear evidence that the fibroblasts in the wound area came from the vicinity of the blood vessels in the peritendon and other areas of loose connective tissue. The observation that the fibrocytes in the tendon away from the wound enlarged, demonstrated nucleoli, and, in fact, came to look like fibroblasts, is confirmed by similar changes occurring in the sclera and Tenon's capsule behind wounds at the limbus of monkeys' eyes (Swan and Ingram, 1965).

Two recent reports from this institution may provide a missing link in correlating these observations. Firstly, Cronin and Jackson (1965) have demonstrated changes in the collagen content of the rat intestine, as much as $1.5 \mathrm{~cm}$. on either side of the wound. Secondly, Weimar (1965) has demonstrated histochemical changes in the corneal cells $3 \mathrm{~mm}$. away from the wound area during the first week after injury, and these changes might have been more widespread if a more severe injury had been inflicted. It is possible that the histological changes noted in the fibrocytes of tendon, sclera, and Tenon's capsule away from the wounds are in some way related to metabolic changes that occur in these tissues on either side of the wound area. Whether or not these cells become fibroblasts is not clear. A view such as this does not necessarily imply that these cells migrate and make a significant contribution to the fibroblasts in the area of the wound.

The formation of adhesions between the tendon and the sclera behind the site of the fold, and between the tendon and Tenon's capsule-in a position occupied by the peritendon in both situations-draws attention to the importance of this layer of loose connective tissue in their formation. Injury to peritendon seems to be a more likely cause of the adhesions than operative bleeding. Haemorrhage was incriminated on the evidence, from tissue culture experiments, that tension forces orientated strands of fibrin in a blood clot (Weiss, 1961), and this guided the migration of fibroblasts between the edges of a wound (Buck, 1953). However, fibrin does not develop into collagen, and there is no evidence to show that the seepage of blood between two uninjured layers of fibrous tissue leads to the formation of adhesions between them. In fact, Gillman, Penn, Bronks, and Roux (1955), studying skin wounds, go so far as to say that blood clot and plasma play no role, other than a protective one, in healing.

Collagen laid down in the organization of a blood clot is absorbed within a few weeks, but it is not resorbed from areas where it seems to serve some purpose, e.g., where it resists some tension forces. This could explain the relatively orderly arrangement of collagen fibres in the two or more strands of fibrous tissue re-attaching the tendon to the sclera after recession of the lateral rectus and re-attachment of the medial rectus. Blood in the wound area may indirectly orientate the fibrous tissue deposited in the first week or so, but the fibrous tissue remaining as adhesions is laid down at a much later stage in the healing process. Such adhesions are more likely to be the result of repair of unnecessary dissection, and measures such as cauterizing minor bleeding points simply damage the tissues, particularly the tendon and peritendon.

The main lesson to be learnt from all these experimental operations is that the surgeon must respect each tissue layer and treat it as a separate entity, for each will heal as such unless the connective tissue between it and its neighbour is disturbed. 
This axiom applies within a given area of the operative field. Extending this area must increase the area of adhesions, and it is clear that the eye is not the place where the surgeon is entitled to give himself wide exposure without having to pay a price.

Lastly, it is my belief that a study of the healing of any particular wound is not complete without an estimate of the rate at which the wound heals. The tensile strength of these wounds has not been measured. However, the absolute rate of deposition of collagen seems to be similar to that found in rabbits (Ingram, 1965b). It is likely, therefore, that the conclusions drawn from that study are, in fact, applicable to man. A fibrous re-attachment was first seen about five days after operation and it was quite definitely formed at ten days. The collagen fibres were immature at this time, but they matured rapidly so that three to four weeks after operation a firm re-attachment had been formed. Thereafter, this fibrous tissue is remodelled.

\section{Summary}

A study has been made of tissue repair after the following operations on the extraocular muscles of the Macaca rhesus monkey: 24 lateral rectus recessions, 24 medial rectus tenotomies and re-attachment, 11 medial rectus recessions combined with excision of Tenon's capsule, and 11 lateral rectus reefings. In contrast to rabbits, a tendon of variable length joins the muscle to the sclera in monkeys, and the results of these operations are more applicable to these same operations in man.

Reference is made to the healing of wounds in tendons elsewhere in the body. The findings support the belief that fibrous tissue deposited between the cut ends of a tendon is laid down by fibroblasts originating in the connective tissue immediately surrounding the tendon. There were, however, changes in the fibrocytes in the tendon, even behind the wound area, and a possible significance of this finding is discussed.

After recession of the lateral rectus, the long tendon of this muscle became joined to the sclera by fibrous tissue, which was closely apposed to the sclera over a wide area extending 2 to $3 \mathrm{~mm}$. in front of the line to which it was sutured. The origin of this fibrous tissue was a thin vascular sheath of connective tissue (peritendon) surrounding the tendon. This is either the episclera or it is continuous with the episclera anteriorly.

The lateral rectus reefing operations showed that extensive damage to this peritendon produced adhesions between the tendon and the tissues on either side of it.

There were two important differences in the process of healing after medial rectus tenotomy and re-attachment: (1) there was no peritendon, because the tendon of the medial rectus was so short; (2) Tenon's capsule, which was opened directly over the wound and not re-sutured, became incorporated in the wound and complicated the healing process.

Study of the healing after the medial rectus recession operations indicated two things: (1) the muscle itself was the source of the fibroblasts in the wound area when muscle or a negligible length of tendon was re-attached to the sclera, whether it was after recession or resection; (2) Tenon's capsule is not necessary to the union of muscle or tendon to sclera. It appears to be relatively inert, as are tendon and sclera. - There seems to be no logical method of restricting the forward extension of the fibrous re-attachment of a tendon to the sclera after recession. The position of the sutures, relative to the other structures in the wound, did not affect this. The 
question of a "pseudotendon", in addition to the true attachment, is raised, and possible causes for its formation are discussed.

Each tissue layer should be regarded as a separate entity, for when each is incised, the edges of the opening have to heal together somehow. The incision in Tenon's capsule should be regarded as another wound, distinct from the underlying tendonto-sclera wound and if the edges gape they will become adherent to other structures because this wound is healed by fibroblasts originating in the episclera. It follows from this that the wider the gap, so the more widespread will be the adhesions. Therefore, minimum opening and precise closure are essential to the restriction of unwanted adhesions that occur after operations for strabismus and detached retina. Operative bleeding is unlikely to cause these adhesions, and rigorous measures to prevent it only damage the tissues unnecessarily.

I am deeply indebted to Dr. Kenneth C. Swan for his help and guidance throughout this investigation, and for giving me the opportunity of spending a year in his department where much of the work was carried out. I also wish to thank Dr. R. P. Burns, Dr. L. Christensen, Dr. D. S. Jackson, and Dr. V. Weimar for their advice over various stages of the work.

The operations were performed at the Oregon Regional Primate Centre. I wish to thank Dr. W. Montagna, Director of this Centre, for permission to use their facilities, and Dr. C. W. de Lannoy for his helpful co-operation throughout. My thanks are also due to all those members of their staffs who looked after the animals before, during, and after the operations.

I wish to thank Mr. T. Booth for preparing the microscopic slides, and Mrs. M. Toney for secretarial help.

This project was financed by Ophthalmology Graduate Training Grant NB-5042, and by the Medical Research Foundation of Oregon. I also gratefully acknowledge a travelling grant from the Wellcome Trust, London.

\section{REFERENCES}

Berens, C. (1938). Amer. J. Ophthal., 21, 536.

Buck, R. C. (1953). J. Path. Bact., 66, 1.

Burns, R. P., and Beighle, R. (1960). Arch. Ophthal. (Chicago), 64, 712.

CARroll, F. D., and BlAKE, E. M. (1932). Ibid., 8, 711.

Chamberlain, W. P. (1954). Trans. Amer. ophthal. Soc., 52, 751.

Cronin, K., and JACKSON, D. S. (1965). Personal communication.

Gallie, W. E., and LeMesurier, A. B. (1924). Brit. J. Surg., 12, 289.

GifFORD, S. R. (1942). Arch. Ophthal., $27,443$.

Gillman, T., Penn, J., Bronks, D., and Roux, M. (1955). Brit. J. Surg., 43, 141.

Grillo, H. C. (1964). Arch. Surg., 88, 218.

HARKNESS, R. D. (1961). Biol. Rev., 36, 399.

Haust, M. D., More, R. H., and Movat, H. Z. (1960). Amer. J. Path., 37, 377.

INGRAM, R. M. (1963). Thesis for D.M. (Oxon.). (1965a). Brit. J. Ophthal., 49, 18. (1965b). Ibid., 49, 235.

Mason, M. L., and Shearon, C. G. (1932). Arch. Surg., 25, 617.

PeER, L. A. (1955). "Transplantation of Tissues", vol. 1, ch. 24, p. 277 . Williams and Wilkins, Baltimore. PIERSE, D. (1962). Trans. ophthal. Soc. U.K., 82, 253.

Romaine, H. H. (1961). Trans. Amer. Acad. Ophthal. Otolaryng., 65, 718.

Sewell, W. R., Wiland, J., and Craver, B. N. (1955). Surg. Gynec. Obstet., 100, 483.

Skoog, T., and Persson, B. H. (1954). Plast. reconstr. Surg., 13, 384.

Swan, K. C. (1956). A.M.A. Arch. Ophthal., 55, 836. and INGRAM, R. M. (1965). To be published. and Talbot, T. (1954). A.M.A. Arch. Ophthal., 51, 32.

Weimar, V. L. (1958). Ibid., 60, 93. (1965). To be published.

Weiss, P. (1961). "The Harvey Lectures 1959-1960”, ser. 55, pp. 13-42. Academic Press, New York.

Whiston, T. B., and Walmsley; R. (1960). J. Bone Jt Surg., 42-B, 377.

Williams, G. (1957). J. Path. Bact., 73, 557.

Worst, J. G. F. (1962). Amer. J. Ophthal., 53, 676.

Young, F. A. (1963). Vision Res., 3, 331. 